TRANSACTIONS OF THE

AMERICAN MATHEMATICAL SOCIETY

Volume 352, Number 6, Pages 2789-2800

S 0002-9947(99)02410-

Article electronically published on December 15, 1999

\title{
PROJECTION ORTHOGONALE SUR LE GRAPHE D'UNE RELATION LINÉAIRE FERMÉ
}

\author{
YAHYA MEZROUI
}

\begin{abstract}
Let $L R(H)$ denote the set of all closed linear relations on a Hilbert space $H$ (which contains all closed linear operators on $H$ ). In this paper, for every $E \in \mathcal{L} R(H)$ we define and study two associated linear operators on $H$, $\cos (E)$ and $\sin (E)$, which play an important role in the study of linear relations. These operators satisfy conditions quite analogous to trigonometric identities (whence their names) and appear, in particular, in the formula that gives the orthogonal projection on the graph of $E$, a formula first established for linear operators by M. H. Stone and extended to linear relations by H. De Snoo. We prove here a slightly modified version of the De Snoo formula. Several other applications of the $\cos (E)$ and $\sin (E)$ operators to operator theory will be given in a forthcoming paper.
\end{abstract}

\section{INTRODUCTION}

Soit $H$ un espace de Hilbert et $H \oplus H$ la somme directe de $H$ avec lui même. $H_{1}$ (resp. $H_{2}$ ) désignera $H \oplus\{0\}$ (resp. $\{0\} \oplus H$ ). On notera $\mathcal{C}(H)$ l'ensemble des opérateurs fermés à domaine dense et $\mathcal{L} R(H)$ l'ensemble des sous-espaces vectoriels fermés de $H \oplus H$, dont les éléments seront appelés relations linéaires. $\mathcal{C}(H)$ s'injecte naturellement dans $\mathcal{L} R(H)$ par l'application qui à un opérateur $A$ fait correspondre son graphe $G(A)$. La topologie la plus utilisée dans $\mathcal{C}(H)$ est celle induite par la métrique du gap $g$ définie par:

$$
g(A, B)=\left\|P_{G(A)}-P_{G(B)}\right\|=\max \left\{\delta\left(P_{G(A)}, P_{G(B)}\right), \delta\left(P_{G(B)}, P_{G(A)}\right)\right\},
$$

où $P_{G(A)}$ (resp. $\left.P_{G(B)}\right)$ désigne la projection orthogonale sur $G(A)$ (resp. sur $G(B)$ ) et $\delta\left(P_{G(A)}, P_{G(B)}\right)=\left\|\left(I-P_{G(B)}\right) P_{G(A)}\right\|$. Le cadre des relations linéaires semble être le bon cadre pour l'étude de la théorie spectrale dans $\mathcal{C}(H)$ (spectre à l'infini) et l'étude des déformations continues d'opérateurs. M.H. Stone [8] a donné pour la première fois la forme explicite de la projection orthogonale sur le graphe d'un élément de $\mathcal{C}(H)$ et $\mathrm{H}$. De Snoo 1 en a donné une extension aux relations linéaires. Cette formule est nécessaire pour l'étude de la métrique du gap sur $\mathcal{L} R(H)$. Dans ce travail, nous donnerons une démonstration de la formule de Stone-De Snoo. Dans ce but nous introduirons d'abord, pour chaque élément $E$ de $\mathcal{L} R(H)$ deux opérateurs associés $\cos (E)$ et $\sin (E)$. Ces opérateurs satisfont des conditions analogues à des identités trigonométriques (d'où leurs noms) et permettent de donner une expression explicite de la projection orthogonale de Stone-De Snoo. En outre, ils permettent d'étendre à $\mathcal{L} R(H)$ un certain nombre de résultats connus dans $\mathcal{C}(H)$ et

Received by the editors February 20, 1998.

1991 Mathematics Subject Classification. Primary 47H06.

Key words and phrases. Orthogonal projection, linear relation, gap metric. 
ils interviennent aussi dans l'étude des déformations continues d'opérateurs comme nous le montrerons dans un autre travail à paraître.

Les principaux résultats de ce travail sont le théorème 1, qui donne la forme explicite de la projection orthogonale sur une relation linéaire, les propositions 10 et 13 qui établissent des identités qui généralisent des identités analogues connues dans le cas des opérateurs et la proposition 14 qui fournit les valeurs des normes et des conormes de $\cos (E)$ et $\sin (E)$ en fonction de $E$.

Notation. Si $E \in \mathcal{L} R(H)$, on notera $E^{\perp}$ son complément orthogonal dans $H \oplus H$. Rappelons quelques définitions données dans [6]: la relation adjointe de $E$, notée $E^{*}$ est définie par:

$$
E^{*}=K\left(E^{\perp}\right) \text { où } K=\left(\begin{array}{rr}
0 & i I \\
-i I & 0
\end{array}\right)
$$

la relation inverse de $E$ notée $E^{-1}$ est définie par:

$$
E^{-1}=J(E) \text { où } J=\left(\begin{array}{cc}
0 & I \\
I & 0
\end{array}\right)
$$

Le composé de deux relations $E$ et $F$ est le sous-espace vectoriel défini par: $E F=$ $\{(x, y) \mid \exists z \in H:(x, z) \in E,(z, y) \in F\}$. La somme de deux relations $E$ et $F$ est le sous-espace vectoriel défini par: $E \hat{+} F=\{(x, y+z) \mid(x, y) \in E,(x, z) \in F\}$ et $\tilde{E}=E \cap\left(E \cap H_{2}\right)^{\perp}$.

Définition 1. Soit $E \in \mathcal{L} R(H)$. On appellera domaine de $E$ et le note $D(E)$ : $D(E)=\{x \in H \mid \exists y \in H$ avec $(x, y) \in E\}$. On appellera noyau de $E$ et on le note $N(E): N(E)=\{x \in H \mid(x, 0) \in E\}$.

On appellera image de $E$ et on le note $R(E)$ :

$$
R(E)=\{y \in H \mid \exists x \in H \text { avec }(x, y) \in E\} .
$$

Soit $\left\{x_{n}\right\}$ une suite de $D(E)$. On dira que $\left\{x_{n}\right\}$ converge vers $x \in D(E)$ pour la norme du graphe $E$ si et seulement si il existe une suite $\left\{y_{n}\right\}$ telle que $\left\{\left(x_{n}, y_{n}\right)\right\}$ $\subseteq E$ et $\left\{\left(x_{n}, y_{n}\right)\right\}$ converge dans $E$. Soit $\left\{y_{n}\right\}$ une suite de $R(E)$. On dira que $\left\{y_{n}\right\}$ converge vers $y \in R(E)$ pour la norme du graphe $E$ si et seulement si il existe une suite $\left\{x_{n}\right\}$ telle que $\left\{\left(x_{n}, y_{n}\right)\right\} \subseteq E$ et $\left\{\left(x_{n}, y_{n}\right)\right\}$ converge dans $E$.

Proposition 1 ([6], proposition 5). Soit $E \in \mathcal{L} R(H)$. Alors $E^{*} E$ est un sousespace vectoriel fermé et par conséquent $G(I) \hat{+} E^{*} E$ l'est aussi.

Preuve. Il est clair que $E^{*} E$ est un sous-espace vectoriel. Montrons qu'il est fermé. Soit $\left\{\left(x_{n}, y_{n}\right)\right\} \subseteq E^{*} E$ une suite qui converge vers $(x, y) \in H \oplus H$, il existe $z_{n} \in H$ tel que $\left(x_{n}, z_{n}\right) \in E$ et $\left(z_{n}, y_{n}\right) \in E^{*}$. D'où $\left(y_{n},-z_{n}\right) \in E^{\perp}$ et on a: $\left\langle x_{n} ; y_{n}\right\rangle-$ $\left\langle z_{n} ; z_{n}\right\rangle=0$, on en déduit alors que la suite $\left\{z_{n}\right\}$ est de Cauchy dans $H$. La suite $\left\{\left(x_{n}, z_{n}\right)\right\}$ est donc convergente dans $E$ et la suite $\left\{\left(z_{n}, y_{n}\right)\right\}$ est convergente dans $E^{*}$. Il existe alors $z \in H$ tel que $(x, z) \in E$ et $(z, y) \in E^{*}$. Par conséquent la suite $\left\{\left(x_{n}, z_{n}\right)\right\}$ converge vers $(x, z) \in E$ et $\left\{\left(z_{n}, y_{n}\right)\right\}$ converge vers $(z, y) \in E^{*}$, car $E$ et $E^{*}$ sont fermés. Donc $(x, y) \in E^{*} E$. Enfin, en utilisant la proposition 5 de [6], on déduit que $G(I) \hat{+} E^{*} E$ est un sous-espace vectoriel fermé.

Lemme 1. Soit $E \in \mathcal{L} R(H)$. Alors:

1) $\forall \varepsilon>0 ; E^{*} E \cap G(-\varepsilon I)=\{0\}$.

2) $E^{*} E \cap H_{2}=E^{*} \cap H_{2}$. 
Preuve. 1) Si $(v,-\varepsilon v) \in E^{*} E$, alors il existe $y \in H$ tel que $(v, y) \in E$ et $(y,-\varepsilon v) \in$ $E^{*}$. Donc $(\varepsilon v, y) \in E^{\perp}$ et on a $\varepsilon\langle v, v\rangle+\langle y, y\rangle=0$. D'où $v=0$.

2) Si $(0, y) \in E^{*}$, alors comme $(0,0) \in E$ on a $(0, y) \in E^{*} E$. Réciproquement si $(0, y) \in E^{*} E$, il existe $z \in H$ tel que $(0, z) \in E$ et $(z, y) \in E^{*}$, d'où $(y,-z) \in E^{\perp}$. Par conséquent $\|z\|^{2}=0 \Longrightarrow z=0$ et $(0, y)=(z, y) \in E^{*}$.

Proposition 2. Soit $E \in \mathcal{L} R(H)$. Alors $J\left(G(I) \hat{+} E^{*} E\right)$ est le graphe d'un opérateur linéaire borné, symétrique et positif. En outre, si on note $\cos (E)$ la racine carré de cet opérateur, alors $N(\cos (E))=D(E)^{\perp}$.

Preuve. Posons $\triangle_{E}=J\left(G(I) \hat{+} E^{*} E\right)$ et soit $(0, v) \in \triangle_{E} \cap H_{2}$ alors $(v, 0) \in$ $G(I) \hat{+} E^{*} E$ donc $(v,-v) \in E^{*} E$, d'où $v=0$ (cf. lemme 11). Par conséquent $\triangle_{E} \cap H_{2}=\{0\}$. Soit maintenant $u \in H$. Comme $H \oplus H=E+E^{\perp}, \exists$ ! $(s, t) \in E$ et $\exists !(x, y) \in E^{\perp}$ tels que:

$$
(u, 0)=(s, t)+(x, y)
$$

et que $\langle x ; s\rangle=-\langle y ; t\rangle$. En outre $(-y, x) \in E^{*}$ et $t=-y$, donc $(s, x) \in E^{*} E$. Par conséquent $(s, u)=(s, s+x) \in G(I) \hat{+} E^{*} E$ et $(u, s) \in \triangle_{E}$, d'où $D\left(\triangle_{E}\right)=H$. On en déduit d'après le théorème du graphe fermé que $\triangle_{E}$ est le graphe d'un opérateur borné, noté $A$ et on a $s=A u$. En outre $\langle u ; A u\rangle=\langle u ; s\rangle=\langle s+x ; s\rangle=$ $\langle s ; s\rangle-\langle y ; t\rangle=\|s\|^{2}+\|t\|^{2}$ d'où

$$
\langle u ; A u\rangle=\langle A u ; u\rangle=\|A u\|^{2}+\|t\|^{2}
$$

ce qui entraîne que $A$ est symétrique, positif et que $\|A\| \leq 1$. En outre $u \in$ $N(\cos (E)) \Longleftrightarrow(0, u) \in\left(G(I) \hat{+} E^{*} E\right) \Longleftrightarrow(0, u) \in E^{*} E \Longleftrightarrow(0, u) \in E^{*} \Longleftrightarrow u \in$ $D(E)^{\perp}$.

Notation. On pose $\nabla_{E}=G(\cos (E))$.

Corollaire 1. Dans l'équation (1), on voit que $x=\cos ^{2}\left(E^{\perp}\right) u$ et par conséquent:

$$
\cos ^{2}(E)+\cos ^{2}\left(E^{\perp}\right)=I .
$$

Lemme 2. Soit $E \in \mathcal{L} R(H)$. Alors: 1) $E^{*} E \subseteq(\tilde{E})^{*} \tilde{E}$. 2) $\cos (E)=\cos (\tilde{E})$.

Preuve. 1) Soit $(a, b) \in E^{*} E$. Alors il existe $d \in H$ tel que $(a, d) \in E$ et $(d, b) \in E^{*}$. Posons $(a, d)=(a, c)+(0, h)$ où $(a, c) \in \tilde{E}$ et $(0, h) \in E \cap H_{2}$. Montrons que $(c, b) \in(\tilde{E})^{*}$, ce qui revient à montrer que $(b,-c) \in(\tilde{E})^{\perp}$. Si $(m, n) \in \tilde{E}$, alors $\langle(b,-d) ;(m, n)\rangle=0$, car $(d, b) \in E^{*}$. En outre: $0=\langle(b,-d) ;(m, n)\rangle=$ $\langle(b,-c-h) ;(m, n)\rangle=\langle(b,-c) ;(m, n)\rangle$, car $(m, n) \in \tilde{E}=E \cap\left(E \cap H_{2}\right)^{\perp}$ et $(0, h) \in E \cap H_{2}$. D'où 1$)$.

2) D'après 1) on a: $E^{*} E \subseteq(\tilde{E})^{*} \tilde{E}$, donc $G(I) \hat{+} E^{*} E \subseteq G(I) \hat{+}(\tilde{E})^{*} \tilde{E}$ et par conséquent: $J\left(G(I) \hat{+} E^{*} E\right) \subseteq J\left(G(I) \hat{+}(\tilde{E})^{*} \tilde{E}\right)$, d'où $\cos ^{2}(E)=\cos ^{2}(\tilde{E})$, ce qui entraîne que $\cos (E)=\cos (\tilde{E})$, par unicité de la racine carré positive.

Proposition 3. Soit $E \in \mathcal{L} R(H)$. Alors:

1) $R\left(\cos ^{2}(E)\right)$ est dense dans $R(\cos (E))$ pour la norme du graphe $G(\cos (E))$.

2) $R(\cos (E)) \subseteq D(E)$.

3) $R\left(\cos ^{2}(E)\right)$ est dense dans $D(E)$ pour la norme du graphe $\tilde{E}$.

4) Soient $E \in \mathcal{L} R(H), v \in D(E)$ et $w$ tels que $(\cos (E) v, w) \in \tilde{E}$. Alors on $a$ :

$$
\|v\|^{2}=\|\cos (E) v\|^{2}+\|w\|^{2} .
$$

5) $R(\cos (E))$ est fermé dans $D(E)$ pour la norme du graphe $\tilde{E}$. 
Preuve. 1) Posons $B=\cos (E)$.

Soit $w \perp N(B)$ et $B w=u \perp R\left(B^{2}\right)$ pour le produit scalaire défini sur le graphe de $B$. Soit $v \in H$ tel que: $0=\langle w ; B v\rangle+\left\langle B w ; B^{2} v\right\rangle=\left\langle w+B^{2} w ; B v\right\rangle \Longrightarrow w+$ $B^{2} w \in R^{\perp}(B)=N(B)$. D'où $B w+B^{3} w=0 \Longrightarrow u+B^{2} u=0 \Longrightarrow\left\langle u+B^{2} u ; u\right\rangle=$ 0 . D'où $\|u\|^{2}+\|B u\|^{2}=0$ et donc $u=0$.

2) Soit $B u \in R(B)$ avec $u \perp N(B)$. $R\left(B^{2}\right)$ est dense dans $R(B)$ d'après 1$)$. Donc il existe une suite $\left\{u_{n}\right\} \subseteq N^{\perp}(B)$ telle que $\left\{B^{2} u_{n}\right\}$ converge vers $B u$ pour la norme du graphe. On en déduit que les suites $\left\{B u_{n}\right\}$ et $\left\{B^{2} u_{n}\right\}$ sont de Cauchy dans $H$. Soit $\left(u_{n}, 0\right)=\left(s_{n}, t_{n}\right)+\left(x_{n}, y_{n}\right)$ la décomposition donnée par l'équation (1). On a alors:

$$
\left\langle B u_{n} ; B u_{n}\right\rangle=\left\|B^{2} u_{n}\right\|^{2}+\left\|t_{n}\right\|^{2}
$$

ce qui prouve que la suite $\left\{t_{n}\right\}$ est de Cauchy dans $H$. En outre comme $s_{n}=B^{2} u_{n}$, la suite $\left\{s_{n}\right\}$ est aussi de Cauchy. Donc la suite $\left\{\left(s_{n}, t_{n}\right)\right\} \subseteq E$ est convergente vers $(s, t) \in E$ et par conséquent $B u=s \in D(E)$.

$3)$ Soit $u \in D(E)$ tel que $\forall v \in H ;\left\langle u ; \cos ^{2}(E) v\right\rangle_{D(E)}=0$. Il existe un unique $(w, z) \in H \times H ;(u, w)$ et $\left(\cos ^{2}(E) v, z\right) \in \tilde{E}$. Soit $(v, 0)=(s, t)+(x, y)$ la décomposition donnée par (11). On a $\left\langle u ; \cos ^{2}(E) v\right\rangle+\langle w ; z\rangle=0$. Or $\langle w ; z\rangle=$ $\langle u ; x\rangle$. En effet: Comme $s=\cos ^{2}(E) v$, alors: $(v-x, v) \in G(I) \hat{+} E^{*} E \Longleftrightarrow(v, v-$ $x) \in \triangle_{E} \Longrightarrow(v-x, x) \in E^{*} E$. En outre comme $E^{*} E \subseteq(\tilde{E})^{*} \tilde{E}$ (cf. lemme 2), alors $(v-x, v) \in(\tilde{E})^{*} \tilde{E}$, d'où il existe $r \in H:(v-x, r) \in \tilde{E},(r, x) \in(\tilde{E})^{*}$. Or $(v-x, z) \in \tilde{E} \Longrightarrow r=z$. Enfin $(z, x)=(r, x) \in(\tilde{E})^{*} \Longrightarrow(-x, z) \in(\tilde{E})^{\perp} \Longrightarrow$ $\langle(u, w) ;(-x, z)\rangle=0 \Longrightarrow\langle w ; z\rangle=\langle u ; x\rangle$, d'où $\left\langle u ; \cos ^{2}(E) v\right\rangle_{D(E)}=0$ ce qui donne: $\left\langle u ; \cos ^{2}(E) v\right\rangle+\langle u ; x\rangle=0 \Longrightarrow\left\langle u ; \cos ^{2}(E) v+x\right\rangle=\langle u ; v\rangle=0$. On en déduit que $u=0$. D'où 3$)$.

4) Soit $u \in H$ et $(u, 0)=(s, t)+(x, y)$ la décomposition donnée par (1) où $E$ est remplacé par $\tilde{E}$. Alors: $s=\cos ^{2}(E) u$ et $\|\cos (E) u\|^{2}=\left\|\cos ^{2}(E) u\right\|^{2}+\|t\|^{2}$. Comme $R(\cos (E))$ est dense dans $D(E)$, il existe $\left\{u_{n}\right\} \subseteq N^{\perp}(\cos (E))$ telle que la suite $\left\{\cos (E) u_{n}\right\}$ converge vers $v$ pour la norme du graphe. En outre il existe une unique suite $\left\{w_{n}\right\}$ telle que $\left(\cos (E) u_{n}, w_{n}\right) \in \tilde{E}$ et on a: $\left(u_{n}, 0\right)=\left(\cos ^{2}(E) u_{n}, w_{n}\right)+$ $\left(x_{n}, y_{n}\right)$ et $\left\|\cos (E) u_{n}\right\|^{2}=\left\|\cos ^{2}(E) u_{n}\right\|^{2}+\left\|w_{n}\right\|^{2}$. Par conséquent $\|v\|^{2}=$ $\|\cos (E) v\|^{2}+\|w\|^{2}$.

5) Montrons d'abord l'égalité suivante.

$$
\forall u \in \overline{D(E)},(\cos (E) u, z) \in \tilde{E} \Longrightarrow\|u\|^{2}=\|\cos (E) u\|^{2}+\|z\|^{2} .
$$

En effet, il existe une suite $\left\{u_{n}\right\} \subseteq D(E)$ qui converge vers $u$ et si on note $\left\{z_{n}\right\}$ l'unique suite telle que $\left(\cos (E) u_{n}, z_{n}\right) \in \tilde{E}$, alors on a: $\left\|u_{n}\right\|^{2}=\left\|\cos (E) u_{n}\right\|^{2}+$ $\left\|z_{n}\right\|^{2}$ et comme $\cos (E)$ est borné, on en déduit que la suite $\left\{z_{n}\right\}$ est convergente. Enfin, par passage à la limite, on obtient l'égalité annoncée.

Montrons maintenant 5). Soient $\left\{\cos (E) u_{n}\right\}$ une suite qui converge vers $v \in$ $D(E)$ pour la norme du graphe $\tilde{E}$ et $\left\{u_{n}\right\} \subseteq \overline{D(E)},\left\{z_{n}\right\}$ telles que $\left(\cos (E) u_{n}, z_{n}\right) \in$ $\tilde{E}$ et $z$ tel que $(v, z) \in \tilde{E}$. Alors (15) donne: $\left\|u_{n}\right\|^{2}=\left\|\cos (E) u_{n}\right\|^{2}+\left\|z_{n}\right\|^{2}$. Comme la suite $\left\{z_{n}\right\}$ converge vers $z$, on en déduit que la suite $\left\{u_{n}\right\}$ est convergente vers un élément $u \in H$, d'où $v=\cos (E) u \in R(\cos (E))$.

Remarque 1 . De 3) et 5) de la proposition 3 , on déduit que:

$$
D(E)=R(\cos (E)) \text {. }
$$


Proposition 4. $E \nabla_{E} \cap\left(E \nabla_{E} \cap H_{2}\right)^{\perp}=E \nabla_{E} \cap\left(E \cap H_{2}\right)^{\perp}$ est le graphe d'un opérateur linéaire borné noté $\sin (E)$.

Preuve. Comme $R(\cos (E))=D(E)$ (cf. remarque 1 ) et $\cos (E)$ est un opérateur borné, alors $E \nabla_{E}$ est un sous-espace vectoriel fermé dont le domaine est tout $H$ (cf. proposition 5 de [6] $) . D\left(E \nabla_{E}\right)=H$, donc $E \nabla_{E} \cap\left(E \nabla_{E} \cap H_{2}\right)^{\perp}$ est le graphe d'un élément de $\mathcal{B}(H)$. Enfin on a $E \nabla_{E} \cap H_{2}=E \cap H_{2}$. En effet: si $(0, y) \in E$, alors $(0, y) \in E \nabla_{E}$ car $(0,0) \in \nabla_{E}$. Réciproquement, si $(0, y) \in E \nabla_{E}$, alors $\exists z \in H$ tel que $(0, z) \in \nabla_{E}$ et $(z, y) \in E$. Mais $(0, z) \in \nabla_{E} \Longrightarrow z=0$, car $\cos (E)$ est un opérateur. D'où $(0, y)=(z, y) \in E$.

Proposition 5. Soit u, $z \in H$ et $(u, 0)=(s, t)+(x, y)$ la décomposition donnée par (11). Alors: 1) $(s, t) \in E \cap\left(E \cap H_{2}\right)^{\perp}$ et $\sin (E) \cos (E) u=t$ 2) $(\cos (E) z, \sin (E) z) \in$ $E \cap\left(E \cap H_{2}\right)^{\perp}$ 3) $N(\cos (E)) \subseteq N(\sin (E))$ 4) $\sin (E)=\sin (\tilde{E})$ 5) $N(\sin (E))=$ $N(\cos (E))+N(E)$.

Preuve. 1) On a $(u, 0)=\left(\cos ^{2}(E) u, t\right)+(x, y)$ avec $(x, y) \in E^{\perp}$ et $t=-y$. $\left(\cos (E) u, \cos ^{2}(E) u\right) \in \nabla_{E}$ et $\left(\cos ^{2}(E), t\right) \in E$, donc $(\cos (E) u, t) \in E \nabla_{E}$. Reste à vérifier que $(\cos (E) u, t) \in\left(E \cap H_{2}\right)^{\perp}$. En effet, si $(0, h) \in E$, alors: $\langle t ; h\rangle=$ $\langle-y ; h\rangle=\langle(-x,-y) ;(0, h)\rangle_{H \oplus H}=0$. On en déduit que $t=\sin (E) \cos (E) u$ et donc que $(s, t) \in E \cap\left(E \cap H_{2}\right)^{\perp}$.

2) $(z, \sin (E) z) \in G(\sin (E))=E \nabla_{E} \cap\left(E \cap H_{2}\right)^{\perp}$ et donc il existe $r \in H$ tel que $(z, r) \in \nabla_{E}$ et $(r, \sin (E) z) \in E$, d'où $r=\cos (E) z$ et on a: $(\cos (E) z, \sin (E) z)=$ $(r, \sin (E)) \in E$. Enfin, il est clair que $(\cos (E) z, \sin (E) z) \in\left(E \cap H_{2}\right)^{\perp}$. D'où $(\cos (E) z, \sin (E) z) \in E \cap\left(E \cap H_{2}\right)^{\perp}$. 0 .

3) Soit $z \in N(\cos (E))$, alors $(0, \sin (E) z) \in\left(E \cap H_{2}\right) \cap\left(E \cap H_{2}\right)^{\perp}$, d'où $\sin (E) z=$

4) Pour montrer que $\sin (E)=\sin (\tilde{E})$, il suffit d'établir que $G(\sin (E)) \subseteq$ $G(\sin (\tilde{E}))=\tilde{E} \nabla_{\tilde{E}}=\tilde{E} \nabla_{E}$. Si $(x, y) \in G(\sin (E))=E \nabla_{E} \cap\left(E \cap H_{2}\right)^{\perp}$, alors $(x, y) \in E \nabla_{E}$ et par conséquent il existe $z \in H$ tel que $(x, z) \in \nabla_{E}$ et $(z, y) \in E$. Or $(x, y) \in\left(E \cap H_{2}\right)^{\perp}$, donc $(z, y) \in\left(E \cap H_{2}\right)^{\perp}$ et $(z, y) \in \tilde{E}$, ce qui entraîne que $(x, y) \in \tilde{E} \nabla_{E}=G(\sin (\tilde{E}))$.

5) Comme $\sin (E)=\sin (\tilde{E})$, alors $N(\sin (E))=N(\sin (\tilde{E}))$. Or $N(\sin (\tilde{E}))=$ $N\left(\nabla_{E}\right)+\left(\left.\cos (E)\right|_{N^{\perp}(\cos (E))}\right)^{-1}\left[R\left(\nabla_{E}\right) \cap N(E)\right]=N\left(\nabla_{E}\right)+N(E)$, car $R\left(\nabla_{E}\right)=$ $D(E)$.

Proposition 6. Soit $E \in \mathcal{L} R(H)$. Alors $\triangle_{E^{*}} E$ est le graphe d'un opérateur linéaire fermable de domaine $D(E)$.

Preuve. Soit $(0, z) \in \triangle_{E^{*}} E$. Alors $\exists t \in H$ tel que $(0, t) \in E$ et $(t, z) \in \triangle_{E^{*}}$, d'où $(0, t) \in E E^{*}$ et $(z, t) \in G(I) \hat{+} E E^{*}$. Donc $(z, t-z) \in E E^{*}$ et par conséquent $(z,-z) \in E E^{*}$. On en déduit que $z=0$ (cf. lemme 1). Pour montrer que $\triangle_{E^{*}} E$ est fermable, il suffit de montrer que $\forall y \neq 0, \in H:(0, y) \notin \overline{\triangle_{E^{*}} E}$, (cf. le iii) du théorème II.2.10 de [2]). Soient $\left\{x_{n}\right\}$ une suite qui converge vers zéro et $\left\{y_{n}\right\}$ une suite qui converge vers un élément $h \in H$. Alors: $\exists z_{n} \in H$ tel que $\left(x_{n}, z_{n}\right) \in E$ et $\left(z_{n}, y_{n}\right) \in \triangle_{E^{*}} \Longrightarrow \exists t_{n} \in H$ tel que:

$$
\left\{\begin{array} { l } 
{ ( x _ { n } , z _ { n } ) \in E } \\
{ ( y _ { n } , t _ { n } ) \in E ^ { * } \text { et } ( t _ { n } , z _ { n } - y _ { n } ) \in E }
\end{array} \Longrightarrow \left\{\begin{array}{l}
\left\langle t_{n} ; x_{n}\right\rangle-\left\langle y_{n} ; z_{n}\right\rangle=0 \\
\left\|t_{n}\right\|^{2}+\left\|y_{n}\right\|^{2}-\left\langle y_{n} ; z_{n}\right\rangle=0 .
\end{array}\right.\right.
$$


De la dernière équation, on tire que

$$
\left\|t_{n}\right\|^{2}+\left\|y_{n}\right\|^{2} \leq\left\|t_{n}\right\|^{2}+\frac{\left\|x_{n}\right\|^{2}}{4} \Longrightarrow\left\|y_{n}\right\|^{2} \leq \frac{\left\|x_{n}\right\|^{2}}{4} .
$$

Alors $\left\{y_{n}\right\}$ converge vers zéro et par conséquent $y=0$.

Lemme 3. Soit $E \in \mathcal{L} R(H)$. Alors:

$$
E\left(G(I) \hat{+} E^{*} E\right)=\left(G(I) \hat{+} E E^{*}\right) E=E \hat{+} E E^{*} E .
$$

Preuve. a) Montrons que $E\left(G(I) \hat{+} E^{*} E\right) \subseteq E \hat{+} E E^{*} E$. Soit $(u, v) \in E\left(G(I) \hat{+} E^{*} E\right)$. Alors $\exists w \in H$ tel que $(u, w) \in G(I) \hat{+} E^{*} E$ et $(w, v) \in E$, d'où $(u, w-u) \in E^{*} E$ et par conséquent $\exists t \in H$ tel que $(u, t) \in E$ et $(t, w-u) \in E^{*}$. On en déduit que $(w-u, v-t) \in E$ et donc que: $(t, v-t) \in E E^{*}$, d'où $(u, v-t) \in E E^{*} E$. En outre $(u, v)=(u, v-t+t)$ avec: $(u, t) \in E$, et $(u, v-t) \in E E^{*} E \Longrightarrow(u, v) \in E \hat{+} E E^{*} E$.

b) Montrons que $\left(G(I) \hat{+} E E^{*}\right) E \subseteq E \hat{+} E E^{*} E$. Soit $(u, v) \in\left(G(I) \hat{+} E E^{*}\right) E$. Alors $\exists w \in H$ tel que

$$
\left\{\begin{array}{l}
(u, w) \in E \\
(w, v) \in G(I) \hat{+} E E^{*} .
\end{array}\right.
$$

d'où $(w, v-w) \in E E^{*}$ ce qui entraîne que $\exists t \in H$

$$
\left\{\begin{array}{l}
(w, t) \in E^{*} \\
(t, v-w) \in E .
\end{array}\right.
$$

Par conséquent $(u, t) \in E^{*} E$ et $(u, v-w) \in E E^{*} E$ et comme $(u, v)=(u, v-w+w)$, alors $(u, v) \in E \hat{+} E E^{*} E$.

c) Montrons que $E \hat{+} E E^{*} E \subseteq E\left(G(I) \hat{+} E^{*} E\right)$. Soit $(x, f+g) \in E \hat{+} E E^{*} E$, avec $(x, f) \in E$ et $(x, g) \in E E^{*} E$. Il existe alors $t \in H$ tel que $(x, t) \in E,(t, s) \in$ $E^{*}$ et $(s, g) \in E$ et on a $(x, s) \in E^{*} E \Longrightarrow(x, s+x) \in G(I) \hat{+} E^{*} E$. En outre $(x, f)$ et $(s, g) \in E \Longrightarrow(x+s, f+g) \in E \Longrightarrow(x, f+g) \in E\left(G(I) \hat{+} E^{*} E\right)$ et donc $E \hat{+} E E^{*} E \subseteq E\left(G(I) \hat{+} E^{*} E\right)$.

d) Montrons que $E \hat{+} E E^{*} E \subseteq\left(G(I) \hat{+} E E^{*}\right) E$. Soit $(x, f+g) \in E \hat{+} E E^{*} E$ tel que $(x, f) \in E$ et $(x, g) \in E E^{*} E$. Il existe alors $t, s \in H$ tels que $(x, t) \in E,(t, s) \in E^{*}$ et $(s, g) \in E$. Donc $(t, g) \in E E^{*} \Longrightarrow(t, g+t) \in G(I) \hat{+} E E^{*} \Longrightarrow(x, g+t) \in$ $\left(G(I) \hat{+} E E^{*}\right) E$. Or $(0, f-t) \in E$ car $(x, f) \in E$ et $(x, t) \in E$ et on a $(0, f-t) \in$ $G(I) \hat{+} E E^{*}$. Alors $(t, f+g) \in G(I) \hat{+} E E^{*}$. En outre $(x, t) \in E$, d'où $(x, f+$ $g) \in\left(G(I) \hat{+} E E^{*}\right) E$. On conclut alors que $E\left(G(I) \hat{+} E^{*} E\right)=\left(G(I) \hat{+} E^{*} E\right) E=$ $E \hat{+} E E^{*} E$.

Lemme 4. Soit $E \in \mathcal{L} R(H)$. Alors $\triangle_{E^{*}}\left(G(I) \hat{+} E E^{*}\right) \subseteq G(I)$.

Preuve. Soit $(u, v) \in \triangle_{E^{*}}\left(G(I) \hat{+} E E^{*}\right)$. Alors $\exists w \in H$ tel que $(u, w) \in G(I) \hat{+} E E^{*}$ et

$$
\begin{aligned}
(w, v) \in \triangle_{E^{*}} & \Longrightarrow\left\{\begin{array} { l } 
{ ( u , w - u ) \in E E ^ { * } } \\
{ ( v , w ) \in G ( I ) \hat { + } E E ^ { * } }
\end{array} \Longrightarrow \left\{\begin{array}{c}
(u, w-u) \in E E^{*} \\
(v, w-v) \in E E^{*}
\end{array}\right.\right. \\
& \Longrightarrow(u-v, v-u) \in E E^{*} \Longrightarrow u=v
\end{aligned}
$$

(cf. 1) du lemme1).

Lemme 5. Soit $E \in \mathcal{L} R(H)$. Alors $G(I) \subseteq\left(G(I) \hat{+} E^{*} E\right) \triangle_{E}$.

Preuve. Si $u \in H$, alors $\exists w \in H$ tel que: $(u, w) \in \triangle_{E} \Longrightarrow(w, u) \in\left(G(I) \hat{+} E^{*} E\right)$ $\Longrightarrow(u, u) \in\left(G(I) \hat{+} E^{*} E\right) \triangle_{E}$. 
Lemme 6. Soit $E \in \mathcal{L} R(H)$. Alors $\triangle_{E^{*}} E \subseteq E \triangle_{E}$.

Preuve. On a $\triangle_{E^{*}} E \subseteq \triangle_{E^{*}} E\left(G(I) \hat{+} E^{*} E\right) \triangle_{E}$ (cf. lemme 5). Or $E\left(G(I) \hat{+} E^{*} E\right)$ $=\left(G(I) \hat{+} E E^{*}\right) E$ (cf. lemme 3). Par conséquent $\triangle_{E^{*}} E \subseteq \triangle_{E^{*}}\left(G(I) \hat{+} E E^{*}\right) E \triangle_{E}$ et on en déduit que $\triangle_{E^{*}} E \subseteq E \triangle_{E}$ (cf. lemme 4).

Proposition 7. Soit $E \in \mathcal{L} R(H)$. Alors $\nabla_{E^{*}} E \subseteq E \nabla_{E}$.

Preuve. Notons $B$ l'opérateur de graphe $\triangle_{E}$ Soient $A$ l'opérateur de graphe $\triangle_{E^{*}}$ et $\left\{Q_{n}\right\}$ une suite de polynômes en $A$ telle que $\lim _{n \rightarrow+\infty} Q_{n}(A)=\cos \left(E^{*}\right)$ dans $\mathcal{B}(H)$ (cf. la proposition 0.5 de [5]). Si $(x, z) \in \nabla_{E^{*}} E$, alors il existe $y \in H$ tel que $(x, y) \in E$ et $(y, z) \in \nabla_{E^{*}}$. On a d'une part $\lim _{n \rightarrow+\infty} Q_{n}(A) y=z$ et d'autre part $\left(y, Q_{n}(A) y\right) \in G\left(Q_{n}(A)\right)$. Or $G\left(Q_{n}(A)\right) E \subseteq E G\left(Q_{n}(B)\right) \Longrightarrow\left(x, Q_{n}(A) y\right) \in$ $E G\left(Q_{n}(B)\right)$. Il existe donc $w_{n} \in H$ tel que

$$
\left\{\begin{array}{l}
\left(x, w_{n}\right) \in G\left(Q_{n}(B)\right) \\
\left(w_{n}, Q_{n}(A) y\right) \in E
\end{array}\right.
$$

d'où $w_{n}=Q_{n}(B) x$ et par conséquent $\lim _{n \rightarrow+\infty} w_{n}=\cos (E) x$. Alors $(\cos (E) x, z) \in E$ entraîne que $(x, z) \in E \nabla_{E}$.

Proposition 8. Soit $E \in \mathcal{L} R(H)$. Alors $\sin \left(E^{*}\right)=[\sin (E)]^{*}$.

Preuve. Montrons que $G\left(\left[\sin \left(E^{*}\right)\right]^{*}\right) \subseteq G(\sin (E))=E \nabla_{E} \cap\left(E \cap H_{2}\right)^{\perp}$. $\left[\sin \left(E^{*}\right)\right]^{*}$ est l'opérateur de graphe: $\left(E^{*} \nabla_{E^{*}} \cap\left(E^{*} \cap H_{2}\right)^{\perp}\right)^{*}=K\left(E^{*} \nabla_{E^{*}}\right)^{\perp}+E^{\perp} \cap H_{1}$.

a) Il est évident que $E^{\perp} \cap H_{1} \subseteq\left(E \cap H_{2}\right)^{\perp}$.

b) $E^{\perp} \cap H_{1} \subseteq E \nabla_{E}$, en effet: soit $(x, 0) \in E^{\perp} \cap H_{1} \Longrightarrow(0, x) \in E^{*} \Longrightarrow(0, x) \in$ $E^{*} E \Longrightarrow(0, x) \in G(I) \hat{+} E^{*} E \Longrightarrow(x, 0) \in \triangle_{E} \Longrightarrow(x, 0) \in \nabla_{E} \Longrightarrow(x, 0) \in E \nabla_{E}$.

c) Montrons que $K\left[\left(E^{*} \nabla_{E^{*}}\right)^{\perp}\right] \subseteq\left(E \cap H_{2}\right)^{\perp}$ Or $K\left[\left(E^{*} \nabla_{E^{*}}\right)^{\perp}\right] \subseteq\left(E \cap H_{2}\right)^{\perp} \Longleftrightarrow$ $K\left(E \cap H_{2}\right) \subseteq E^{*} \nabla_{E^{*}}$ Soit donc $(0, f) \in\left(E \cap H_{2}\right)$, alors $(0, f) \in E E^{*} \Longrightarrow(0, f) \in$ $G(I) \hat{+} E E^{*}$. On en déduit que $(f, 0) \in \triangle_{E^{*}}$ et donc que $K[(0, f)]=(f, 0) \in \nabla_{E^{*}}$, d'où: $(f, 0) \in E^{*} \nabla_{E^{*}}$.

d) Montrons que $\left(E^{*} \nabla_{E^{*}}\right)^{*} \subseteq E \nabla_{E}$ Ceci équivaut à dire que $\left(E^{*} \nabla_{E^{*}}\right)^{*} \subseteq$ $\left(\nabla_{E} E^{*}\right)^{*} \Longleftrightarrow \nabla_{E} E^{*} \subseteq E^{*} \nabla_{E^{*}}$, ce qui est vrai (cf. proposition 7 ).

On vient de montrer que $\left(\sin \left(E^{*}\right)\right)^{*} \subseteq \sin (E)$ et comme $\left[\sin \left(E^{*}\right)\right]^{*}$ est un opérateur borné, on en déduit que $\left[\sin \left(E^{*}\right)\right]^{*}=\sin (E)$.

Proposition 9. Soit $E \in \mathcal{L} R(H)$. Alors $\nabla_{E^{*}} E \subseteq G(\sin (E)$ ) (c'est à dire que l'opérateur qui a pour graphe le sous-espace vectoriel $\nabla_{E^{*}} E$ est la restriction de l'opérateur $\sin (E)$ à $D(E)$ ).

Preuve. $\cos \left(E^{*}\right)$ est un opérateur symétrique, donc $\left(\nabla_{E^{*}}\right)^{*}=\nabla_{E^{*}}$ et on peut donc écrire que $\nabla_{E^{*}} E=\left(\nabla_{E^{*}}\right)^{*}\left(E^{*}\right)^{*} \subseteq\left(E^{*} \nabla_{E^{*}}\right)^{*}$ (cf. proposition 10 de [6]). Donc $\nabla_{E^{*}} E \subseteq\left\{G\left(\sin \left(E^{*}\right)\right)\right\}^{*}=G(\sin (E))$, d'où on voit que $\sin (E)$ est une extension de l'opérateur dont le graphe est $\nabla_{E^{*}} E$.

Proposition 10. Soit $E \in \mathcal{L} R(H)$ et $P_{N(\cos (E))}$ la projection orthogonale sur $N(\cos (E))$. Alors:

$$
\cos ^{2}(E)+\sin \left(E^{*}\right) \sin (E)=I-P_{N(\cos (E))}
$$

ce qui justifie les notations $\cos$ et $\sin$. 
Preuve. Soit $u \in H$, on pose $C=\cos ^{2}(E)+\sin \left(E^{*}\right) \sin (E)$ et $v=\cos (E) u$. Si $(u, 0)=(s, t)+(x, y)$ est la décomposition donnée par (1), alors $(v, t) \in E \nabla_{E}$. En outre $t=\sin (E) \cos (E) u$ (cf. Proposition [5) et comme $R(\cos (E))=D(E)$ en utilisant (2) et la proposition 8 , on voit que:

$$
\forall v \in \overline{D(E)}\left\langle v ;\left(\cos ^{2}(E)+\sin \left(E^{*}\right) \sin (E)\right) v\right\rangle=\|v\|^{2} .
$$

Soit maintenant $w \in H$ et $v=P_{\overline{D(E)}} w$. Alors:

$$
\langle w ; C w\rangle=\left\langle P_{N(\cos (E))} w+P_{\overline{D(E)}} w ; C w\right\rangle=\left\langle P_{N(\cos (E))} w ; C P_{\overline{D(E)}} w\right\rangle .
$$

En utilisant (7) on trouve:

$$
\langle w ; C w\rangle=\left\langle w ; P_{\overline{D(E)}} w\right\rangle \Longrightarrow\left\langle w ;\left(C-P_{\overline{D(E)}}\right) w\right\rangle=0
$$

Par polarisation on en déduit que $C-P \overline{D(E)}=0$, d'où: $\cos ^{2}(E)+\sin \left(E^{*}\right) \sin (E)=$ $P_{\overline{D(E)}}=I-P_{N(\cos (E))}$. On va rappeler un résultat connu qui nous sera utile pour la suite.

Proposition 11. Soit $A$ un opérateur fermé à domaine dense et

$$
c(A)=\inf _{x \in D(A), x \in N^{\perp}(A)} \frac{\|A x\|}{\|x\|}
$$

sa conorme (cf. [7], Théorème 5.2, p. 231, où la conorme de A est notée $\gamma(A)$ et appelée "reduced minimum modulus of $A$ "). Alors:

$$
c\left(A A^{*}\right)=c\left(A^{*} A\right)=c(A) c\left(A^{*}\right)=c^{2}(A) .
$$

Proposition 12. Soit $E \in \mathcal{L} R(H)$. Alors: 1) $\|\sin (E)\|^{2}+c^{2}(\cos (E))=1$,

2) $\left\|\left.\cos (E)\right|_{N^{\perp}(E)}\right\|^{2}+c^{2}(\sin (E))=1$.

Preuve. 1) Si $u \in N^{\perp}(\sin (E)) \subseteq N^{\perp}(\cos (E))$ et $\|u\| \leq 1$, alors d'après l'équation ([6]): $\|\sin (E) u\|^{2}=\|u\|^{2}-\|\cos (E) u\|^{2} \leq\|u\|^{2}-c^{2}(\cos (E))\|u\|^{2}=$ $\left(1-c^{2}(\cos (E))\right)\|u\|^{2}$. Par conséquent: $\|\sin (E)\|^{2} \leq 1-c^{2}(\cos (E))$. Inversement, on peut écrire: $\|\cos (E) u\|^{2}=\|u\|^{2}-\|\sin (E) u\|^{2} \geq\|u\|^{2}-\|\sin (E)\|^{2}\|u\|^{2}$, par conséquent $\|\sin (E)\|^{2} \geq 1-c^{2}(\cos (E))$, d'où 1$)$.

2) Si $u \in N^{\perp}(\sin (E)) \subseteq N^{\perp}(E) \cap N^{\perp}(\cos (E))$, alors d'après l'équation (6) on a: $\|\sin (E) u\|^{2}=\|u\|^{2}-\|\cos (E) u\|^{2} \geq\|u\|^{2}-\left\|\left.\cos (E)\right|_{N^{\perp}(E)}\right\|^{2}\|u\|^{2}$. D'où $c^{2}(\sin (E)) \geq 1-\left\|\left.\cos (E)\right|_{N^{\perp}(E)}\right\|^{2}$. Inversement, comme

$$
\begin{aligned}
N^{\perp}(E) & =N^{\perp}(\sin (E))+\left(N^{\perp}(\cos (E)) \cap N^{\perp}(E)\right)^{\perp} \cap N^{\perp}(E) \\
& =N^{\perp}(\sin (E))+(N(\cos (E))+N(E)) \cap N^{\perp}(E) \\
& =N^{\perp}(\sin (E))+N(\cos (E)) \cap N^{\perp}(E) \\
& =N^{\perp}(\sin (E))+N(\cos (E)) .
\end{aligned}
$$

Soit $u \in N^{\perp}(E)$ et $u=v+w$, où $v \in N^{\perp}(\sin (E))$ et $w \in N(\cos (E))$. En utilisant l'équation (6) , on obtient alors: $\|\cos (E) u\|^{2}=\|u\|^{2}-\|\sin (E) v\|^{2}-\|w\|^{2}$. D'où $\|\cos (E) u\|^{2} \leq\|v\|^{2}-c^{2}(\sin (E))\|v\|^{2} \leq\left(1-c^{2}(\sin (E))\right)\|u\|^{2}$ et par conséquent $\left\|\left.\cos (E)\right|_{N^{\perp}(E)}\right\|^{2} \leq 1-c^{2}(\sin (E))$, d'où 2$)$.

Remarque 2. Soit $E \in \mathcal{L} R(H)$. Alors $\|\sin (E)\| \leq 1$.

Proposition 13. Soit $E \in \mathcal{L} R(H)$. Alors $\cos \left(E^{*}\right) \sin (E)=\sin (E) \cos (E)$. 
Preuve. Montrons d'abord que $G\left(\cos \left(E^{*}\right) \sin (E)\right) \subseteq E \nabla_{E} \nabla_{E}$. Soit $(x, z) \in$ $G\left(\cos \left(E^{*}\right) \sin (E)\right)$. Il existe $y \in H$ tel que:

$$
\left\{\begin{array}{l}
(x, y) \in G(\sin (E)) \\
(y, z) \in G\left(\cos \left(E^{*}\right)\right) .
\end{array}\right.
$$

$1^{\text {er }}$ cas) Si $x \in D(E)$, alors $(x, y) \in \nabla_{E^{*}} E$ (cf. proposition 9) et on en déduit que $(x, z) \in \nabla_{E^{*}} \nabla_{E^{*}} E \subseteq E \nabla_{E} \nabla_{E}$ (cf. lemme 6).

$2^{\text {eme }}$ cas) Si $x$ est un élément quelconque de $H$, on écrit: $x=x_{1}+x_{2}$ avec $x_{1} \in$ $\overline{D(E)}$ et $x_{2} \in D(E)^{\perp} x_{2} \in D(E)^{\perp} \Longrightarrow\left(x_{2}, 0\right) \in \nabla_{E}$ (cf. proposition 2). $\Longrightarrow$ $\left(x_{2}, 0\right) \in E \nabla_{E} \Longrightarrow\left(x_{2}, 0\right) \in G(\sin (E)) \Longrightarrow\left(x_{1}, y\right) \in G(\sin (E))$. On obtient alors

$$
\left\{\begin{array}{l}
\left(x_{1}, z\right) \in G\left(\cos \left(E^{*}\right) \sin (E)\right) \\
x_{1} \in \overline{D(E)}
\end{array}\right.
$$

et il existe donc une suite $\left\{t_{n}\right\} \subseteq D(E)$ telle que

$$
\lim _{n \rightarrow+\infty}\left(t_{n}, \cos \left(E^{*}\right) \sin (E) t_{n}\right)=\left(x_{1}, z\right)
$$

car $\cos \left(E^{*}\right) \sin (E)$ est un opérateur borné.

En outre $\left(t_{n}, \cos \left(E^{*}\right) \sin (E) t_{n}\right) \in G\left(\cos \left(E^{*}\right) \sin (E)\right)$ avec $t_{n} \in D(E)$. D'où $\left(t_{n}, \cos \left(E^{*}\right) \sin (E) t_{n}\right) \in E \nabla_{E} \nabla_{E}$ (cf. premier cas). Comme $E \nabla_{E} \nabla_{E}$ est fermé, $\left(x_{1}, z\right) \in E \nabla_{E} \nabla_{E}$. Par ailleurs $\left(x_{2}, 0\right) \in G(\cos (E))$ ce qui entraîne que $\left(x_{2}, 0\right) \in$ $E \nabla_{E} \nabla_{E}$. Finalement on conclut que $G\left(\cos \left(E^{*}\right) \sin (E)\right) \subseteq E \nabla_{E} \nabla_{E}$.

Montrons maintenant que

$$
\cos \left(E^{*}\right) \sin (E)=\sin (E) \cos (E)(x, z) \in G\left(\cos \left(E^{*}\right) \sin (E)\right)
$$

$\Longrightarrow(x, z) \in E \nabla_{E} \nabla_{E} \Longrightarrow \exists t \in H$ tel que

$$
\left\{\begin{array}{l}
(x, t) \in G(\cos (E)) \\
(t, z) \in E \nabla_{E}
\end{array}\right.
$$

ce qui revient à montrer que $(t, z) \in\left(E \cap H_{2}\right)^{\perp}$. En effet: $z \in R\left(\cos \left(E^{*}\right)\right)$ et $R\left(\cos ^{2}\left(E^{*}\right)\right)$ est dense dans $R\left(\cos \left(E^{*}\right)\right)$ (cf. proposition 3). Il existe donc une suite $\left\{z_{n}\right\} \subseteq R\left(\cos ^{2}\left(E^{*}\right)\right)$ et une suite $\left\{y_{n}\right\} \subseteq H$ telles que

$$
\lim _{n \rightarrow+\infty} z_{n}=z \text { et }\left(y_{n}, z_{n}\right) \in G\left(\cos ^{2}\left(E^{*}\right)\right) \Longrightarrow\left(z_{n}, y_{n}-z_{n}\right) \in E E^{*} \Longrightarrow \exists s_{n} \in H
$$

tel que:

$$
\left\{\begin{array} { l } 
{ ( z _ { n } , s _ { n } ) \in E ^ { * } } \\
{ ( s _ { n } , y _ { n } - z _ { n } ) \in E }
\end{array} \Longrightarrow \left\{\begin{array}{l}
\left(s_{n},-z_{n}\right) \in E^{\perp} \\
\left(s_{n}, y_{n}-z_{n}\right) \in E .
\end{array}\right.\right.
$$

Soit maintenant $(0, f) \in E$. Alors: $\left\langle\left(s_{n},-z_{n}\right) ;(0, f)\right\rangle=0 \Longrightarrow\left\langle z_{n}, f\right\rangle=0 \Longrightarrow$ $\langle z ; f\rangle=0$.

Lemme 7. Soit $E$ un sous-espace vectoriel fermé et $(x, y) \in E$. Alors: $\exists ! u \in$ $N^{\perp}(\cos (E))$ et $\exists ! v \in N\left(\cos \left(E^{*}\right)\right)$ avec $(x, y)=(\cos (E) u, \sin (E) u+v)$ Réciproquement: $\forall u \in N^{\perp}(\cos (E))$ et $v \in N\left(\cos \left(E^{*}\right)\right) ;(\cos (E) u, \sin (E) u+v) \in E$.

Preuve. $E=E \cap\left(E \cap H_{2}\right)^{\perp}+E \cap H_{2}$. Si $(x, y) \in E$, alors: $\exists(a, b) \in E \cap$ $\left(E \cap H_{2}\right)^{\perp}$ et $(0, v) \in E \cap H_{2}$ tels que $(x, y)=(a, b)+(0, v) R(\cos (E))=D(E) \Longrightarrow$ $\exists ! u \in N^{\perp}(\cos (E))$ tel que $a=\cos (E) u$ et donc $b=\sin (E) \cos (E) u$. En outre $(\cos (E) u, \sin (E) u) \in G(\sin (E))$. En effet: $(u, \sin (E) u) \in G(\sin (E))=E \nabla_{E} \cap$ 
$\left(E \cap H_{2}\right)^{\perp} \Longrightarrow \exists z \in H$ tel que: $(u, z) \in \nabla_{E}$ et $(z, \sin (E) u) \in E \Longrightarrow z=\cos (E) u$ $\Longrightarrow(\cos (E) u, \sin (E) u) \in E .(u, \sin (E) u) \in\left(E \cap H_{2}\right)^{\perp}$, par conséquent

$$
(\cos (E) u, \sin (E) u) \in E \cap\left(E \cap H_{2}\right)^{\perp} .
$$

Théorème 1. Soit $E \in \mathcal{L} R(H)$. Alors la projection orthogonale sur $E$ est donnée par:

$$
P_{E}=\left(\begin{array}{ll}
\cos ^{2}(E) & \cos (E) \sin \left(E^{*}\right) \\
\sin (E) \cos (E) & I-\cos ^{2}\left(E^{*}\right)
\end{array}\right) .
$$

Preuve. De la proposition [10, on déduit que $P_{E}$ est auto-adjoint. Montrons maintenant que $P_{E}(x, y)=(x, y) \Longleftrightarrow(x, y) \in E$

$\Longleftarrow$ Si $(x, y) \in E \Longrightarrow x \in D(E) \Longrightarrow P_{N(\cos (E))} x=0$ et on a: $(x, y)=$ $(\cos (E) u, \sin (E) u+v)$ avec $u \in N^{\perp}(\cos (E))$ et $(0, v) \in E \cap H_{2} P_{E}(x, y)=$ $\left(\cos ^{2}(E) x+\cos (E) \sin \left(E^{*}\right) y, \cos \left(E^{*}\right) \sin (E) x+y-\cos ^{2}\left(E^{*}\right) y\right) \cos ^{2}(E) x+\cos (E)$ $\cdot \sin \left(E^{*}\right) y=x-\sin \left(E^{*}\right) \sin (E) x+\cos (E) \sin \left(E^{*}\right) y$ (cf. Proposition 10$)=x+\sin \left(E^{*}\right)$ - $\left\{\cos \left(E^{*}\right) y-\sin (E) x\right\}=x+\sin \left(E^{*}\right)\left\{\cos \left(E^{*}\right) \sin (E) u-\sin (E) \cos (E) u\right\}=x$ (cf. proposition 13). De même: $\cos \left(E^{*}\right) \sin (E) x+y-\cos ^{2}\left(E^{*}\right) y=y+\cos \left(E^{*}\right)\{\sin (E) x-$ $\left.\cos \left(E^{*}\right) y\right\}=y$.

$$
\begin{aligned}
& \Longrightarrow) \\
& \quad P_{E}(x, y)=(x, y) \Longleftrightarrow\left\{\begin{array}{l}
\cos ^{2}(E) x+\cos (E) \sin \left(E^{*}\right) y=x \\
\cos \left(E^{*}\right) \sin (E) x+y-\cos ^{2}\left(E^{*}\right) y=y .
\end{array}\right.
\end{aligned}
$$

La première équation peut s'écrire: $x=\cos (E)\left\{\cos (E) x+\sin \left(E^{*}\right) y\right\}$. Donc $x \in$ $D(E)$, car $R(\cos (E))=D(E)$. Posons $u=\cos (E) x+\sin \left(E^{*}\right) y$. Il suffit donc de montrer que: $t=y-\sin (E) u \in N\left(\cos \left(E^{*}\right)\right.$ ) (cf. lemme 71). Or $\cos \left(E^{*}\right) t=$ $\cos \left(E^{*}\right)\{y-\sin (E) u\}=\cos \left(E^{*}\right)\left\{y-\sin (E) \cos (E) x-\sin (E) \sin \left(E^{*}\right) y\right\}=\cos \left(E^{*}\right)$. $\left\{\cos ^{2}\left(E^{*}\right) y-\sin (E) \cos (E) x+P_{N\left(\cos \left(E^{*}\right)\right)} y\right\}=\cos \left(E^{*}\right)\left\{\cos ^{2}\left(E^{*}\right) y-\sin (E) \cos (E) x\right\}$. Or de la seconde équation, on tire que:

$$
\cos ^{2}\left(E^{*}\right) y=\cos \left(E^{*}\right) \sin (E) x=\sin (E) \cos (E) x
$$

D'où $\cos \left(E^{*}\right) t=0$, par conséquent: $(x, y)=(\cos (E) u, \sin (E) u+y-\sin (E) u) \in E$.

Remarque 3. Le théorème précédent a d'abord était démontré dans le cas des opérateurs par M. H. Stone [8] et étendu à des relations fermées quelconques par H. De Snoo [1].

Proposition 14. Soit $E \in \mathcal{L} R(H)$. Alors:

1) $\left\|\cos ^{2}(E)\right\|=\delta^{2}\left(E, H_{2}\right)$ et $\|\cos (E)\|=\delta\left(E, H_{2}\right)$.

2) $c\left(\cos ^{2}(E)\right)=1-\varepsilon^{2}\left(E, H_{1}\right)$ et $c(\cos (E))=\sqrt{1-\varepsilon^{2}\left(E, H_{1}\right)}$.

3) $\cos ^{2}(E) P_{N^{\perp}(E)}=\cos ^{2}(F)$, avec $F=E \cap\left(E \cap H_{1}\right)^{\perp}$.

4) $\|\sin (E)\|^{2}=\varepsilon^{2}\left(E, H_{1}\right)$ et $c^{2}(\sin (E))=1-\varepsilon^{2}\left(E, H_{2}\right)$.

Preuve. 1) $\left\|\cos ^{2}(E)\right\|=\left\|P_{1} P_{E} P_{1}\right\|=\left\|P_{1} P_{E} P_{E} P_{1}\right\|=\left\|\left(P_{1} P_{E}\right)\left(P_{1} P_{E}\right)^{*}\right\|=$ $\left\|P_{1} P_{E}\right\|^{2}=\delta^{2}\left(E, H_{2}\right)$. Comme $\cos (E)$ est symétrique, $\|\cos (E)\|=\delta\left(E, H_{2}\right)$.

2) $c^{2}\left(P_{1} P_{E}\right)=c\left(\left(P_{1} P_{E}\right)\left(P_{1} P_{E}\right)^{*}\right)=c\left(P_{1} P_{E} P_{1}\right)=c\left(\cos ^{2}(E)\right)$ (cf. proposition 11). Par ailleurs $\varepsilon^{2}\left(E, H_{1}\right)+c^{2}\left(P_{1} P_{E}\right)=1$ (cf. la proposition 1.2 .2 de [4] ]), donc $c\left(\cos ^{2}(E)\right)=1-\varepsilon^{2}\left(E, H_{1}\right)$ et par conséquent $c(\cos (E))=\sqrt{1-\varepsilon^{2}\left(E, H_{1}\right)}$, puisque $c\left(\cos ^{2}(E)\right)=c(\cos (E)) c(\cos (E))=c^{2}(\cos (E))$ et $\cos (E)$ est symétrique. 
3) On a $E=E \cap H_{1}+F$ et $P_{E}=P_{F}+P_{E \cap H_{1}}$, puisque $E \cap H_{1}$ et $F$ sont orthogonaux. Comme

$$
P_{E \cap H_{1}}=\left(\begin{array}{ll}
P_{N(E)} & 0 \\
0 & 0
\end{array}\right)
$$

alors:

$$
\cos ^{2}(E)=P_{N(E)}+\cos ^{2}(F)
$$

ce qui entraîne que $\cos ^{2}(E) P_{N^{\perp}(E)}=\cos ^{2}(F) P_{N^{\perp}(E)}=\cos ^{2}(F)\left(I-P_{N(E)}\right)=$ $\cos ^{2}(F)$, car $N(E) \subseteq N(\cos (F))$.

4) D'après le 1) de la proposition 12 on a: $\|\sin (E)\|^{2}=1-c^{2}(\cos (E))=$ $\varepsilon^{2}\left(E, H_{1}\right)$. En outre d'après le 2$)$ de la même proposition, on obtient: $c^{2}(\sin (E))=$ $1-\left\|\cos (E) P_{N^{\perp}(E)}\right\|^{2}=1-\left\|\cos ^{2}(E) P_{N^{\perp}(E)}\right\|$. En effet, comme $\cos ^{2}(E) P_{N^{\perp}(E)}=$ $\cos ^{2}(F)$, alors $\cos ^{2}(E) P_{N^{\perp}(E)}$ est symétrique et par conséquent $\cos ^{2}(E) P_{N^{\perp}(E)}=$ $P_{N \perp(E)} \cos ^{2}(E)$. On peut écrire alors

$$
\begin{aligned}
& \left\|\cos (E) P_{N^{\perp}(E)}\right\|^{2}=\left\|\left(\cos (E) P_{N^{\perp}(E)}\right)^{*} \cos (E) P_{N^{\perp}(E)}\right\| \\
& =\left\|P_{N^{\perp}(E)} \cos ^{2}(E) P_{N^{\perp}(E)}\right\| \\
& =\left\|\cos ^{2}(E) P_{N^{\perp}(E)} P_{N^{\perp}(E)}\right\| \\
& =\left\|\cos ^{2}(E) P_{N \perp(E)}\right\| \text {. }
\end{aligned}
$$

Par ailleurs $\varepsilon^{2}\left(E, H_{2}\right)=\left\|P_{1} P_{E \cap\left(E \cap H_{1}\right)} P_{1}\right\|=\left\|\cos ^{2}(F)\right\|$.

Proposition 15. Soit $E \in \mathcal{L} R(H)$. Alors:

$$
P_{\tilde{E}}=P_{E}+\left(\begin{array}{ll}
0 & 0 \\
0 & -P_{N\left(\cos \left(E^{*}\right)\right)}
\end{array}\right) \text {. }
$$

Preuve. $P_{E}=P_{\tilde{E}}+P_{E \cap H_{2}}$, avec

$$
P_{E \cap H_{2}}=\left(\begin{array}{ll}
\cos ^{2}\left(E \cap H_{2}\right) & \cos \left(E \cap H_{2}\right) \sin \left(\left[E \cap H_{2}\right]^{*}\right) \\
\sin \left(E \cap H_{2}\right) \cos \left(E \cap H_{2}\right) & I-\cos ^{2}\left(\left[E \cap H_{2}\right]^{*}\right)
\end{array}\right) .
$$

Or $\cos \left(E \cap H_{2}\right)=0$, donc

$$
P_{E \cap H_{2}}=\left(\begin{array}{ll}
0 & 0 \\
0 & I-\cos ^{2}\left(\left[E \cap H_{2}\right]^{*}\right)
\end{array}\right) .
$$

En outre $I-\cos ^{2}\left(\left[E \cap H_{2}\right]^{*}\right)=P_{N\left(\cos \left(\left[E \cap H_{2}\right]^{*}\right)\right)}=P_{N\left(\cos \left(E^{*}\right)\right)}$. En effet:

$$
\begin{aligned}
x \in N\left(\cos \left(\left[E \cap H_{2}\right]^{*}\right)\right) & \Longleftrightarrow x \in D^{\perp}\left(\left[E \cap H_{2}\right]^{*}\right) \Longleftrightarrow(0, x) \in E \cap H_{2} \\
& \Longleftrightarrow x \in N\left(\cos \left(E^{*}\right)\right) .
\end{aligned}
$$

Remarque 4. 1) On vérifie sans peine que

$$
Q(E)=P_{E}+\left(\begin{array}{ll}
P_{N(\cos (E))} & 0 \\
0 & -P_{N\left(\cos \left(E^{*}\right)\right)}
\end{array}\right)
$$

est une projection orthogonale sur le graphe d'un opérateur fermé à domaine dense, qu'on appelle "opératisé" de $E$. Cette opératisation se ramène à éliminer la partie verticale $E \cap H_{2}$ de la relation et la définir sur un espace dense en l'occurence sur $D(E)+D^{\perp}(E)$, en envoyant $D^{\perp}(E)$ sur zéro. En fait $Q(E)=P_{E}+P_{D^{\perp}(E) \oplus\{0\}}-$ $\mathrm{PE \cap H}_{2}$

2) On se place maintenant dans le cadre des opérateurs. Soit $A \in \mathcal{C}(H)$ et posons $E=J[G(A)]=[G(A)]^{-1}$ l'inverse au sens des relations du graphe de $A$. Alors la 
projection orthogonale sur le graphe de l'inverse de Moore-Penrose de $A$ est donnée par la matrice $Q(E)$. On retrouve les résultats donnés dans [3] (le lemme 2.5, les corollaires 2.6 et 2.8) concernant les relations entre les projections orthogonales d'un opérateur et celle de son inverse de Moore-Penrose:

$$
Q(E)=\left(\begin{array}{ll}
I-\cos ^{2}\left(E^{*}\right)+P_{N\left(E^{*}\right)} & \cos \left(E^{*}\right) \sin (E) \\
\cos (E) \sin \left(E^{*}\right) & \cos ^{2}(E)-P_{N(E)}
\end{array}\right) .
$$

Donc le résultat de "l'opératisation" de la relation $[G(A)]^{-1}$.

\section{REFERENCES}

[1] H. De Snoo, Manuscrit communiqué par l'auteur.

[2] S. Goldberg, Unbounded linear operators, McGraw-Hill, New York, 1966. MR 34:1580

[3] J. P. Labrousse, Inverses généralisés d'opérateurs non-bornés, Proc. Amer. Math. Soc. 115, (1992). MR 92h:47002

[4] J. P. Labrousse, Les opérateurs quasi-Fredholm: une généralisation des opérateurs semiFredholm, Rend. Circ. Mat. Palermo, 29 (1980), 161-258. MR 83c:47022]

[5] J. P. Labrousse \& B. Mercier, Extension du théorème de Brown-Douglas-Fillmore au cas des opérateurs non-bornés, J. Operator Theory, 24 (1990), 137-154. MR 92j:47040

[6] Y. Mezroui, Le complété des opérateurs fermés à domaine dense pour la métrique du gap, J. Operator Theory, 41 (1999), 69-92. CMP 99:09

[7] T. Kato, Perturbation theory for linear operators, Springer-Verlag, Berlin, 1966. MR 34:3324

[8] M. H. Stone, On unbounded operators on a Hilbert space, J. Indian Math. Soc., 15 (1951). MR 14:565d

Laboratoire J.A. Dieudonné, UMR \#6621 du CNRS, Université de NiCe - Sophia ANtipolis, 06108 Nice, Cedex 2, France

E-mail address: mezroui@math.unice.fr 